\title{
Hirayama Disease: Best Depicted in the Prone Position with Full-Flexion Cervical MRI in A Serial Trial
}

\author{
Yu Hui Won (iD) 1,2 and Eun Hae Park (iDi ${ }^{2,3,{ }^{*}}$ \\ ${ }^{1}$ Department of Physical Medicine and Rehabilitation, Research Institute of Clinical Medicine of Chonbuk National University, Chonbuk National University Medical School, \\ Jeonju, Republic of Korea \\ ${ }^{2}$ Biomedical Research Institute of Chonbuk National University Hospital, Jeonju, Republic of Korea \\ ${ }^{3}$ Department of Radiology, Chonbuk National University Medical School, Research Institute of Clinical Medicine of Chonbuk, Jeonju, Republic of Korea \\ "Corresponding author: Department of Radiology, Chonbuk National University Medical School, Jeonju, Republic of Korea. Email: mbgracie@gmail.com
}

Received 2018 June 14; Revised 2018 November 22; Accepted 2018 November 26.

\begin{abstract}
The current case report presents a 17-year-old man newly diagnosed with Hirayama Disease (HD) with right-side upper limb weakness, which was best detected by full cervical flexion scan in the prone position, using a serial trial of flexion Magnetic Resonance Imaging (MRI). Using a neck coil resulted in inadequate flexion angle because of limited space and failed to demonstrate anterior dural displacement, which is known to be the most significant and important finding for the diagnosis of HD. By serial flexion scan, the researchers found that dural displacement may not be evident because of an insufficient flexion angle, and that as the flexion angle increases, the diameter and level of anterior dural displacement increases, emphasizing the necessity of full-flexion imaging in patients with clinically suspicious HD. In the present case, the prone position provided comfortable stabilization for ideal full-flexion position, resulting in optimized image quality.
\end{abstract}

Keywords: Hirayama Disease, Cervical MRI, Prone Position

\section{Introduction}

Hirayama Disease (HD), also known as benign juvenile brachial spinal muscular atrophy, is a pure motor focal amyotrophy distributed in the $\mathrm{C} 7, \mathrm{C} 8$, and $\mathrm{T} 1$ spinal segmental innervated muscles. This rare disease occurs between adolescence and the early third decade of life with male preponderance. The main characteristics of this disease include an insidious onset of unilateral or asymmetric muscular atrophy and weakness in the distal upper limb (13).

The forward displacement of the posterior cervical dural sac, resulting in flattening of the lower cervical cord when the neck is in flexion, is a characteristic of the disease. Gourie-Devi et al. termed it "monomelic amyotrophy," which belonged to the category of localized motor neuron diseases (4). However, HD is postulated to be a lower cervical myelopathy during neck flexion because the dura cannot sufficiently compensate the longer length of the cervical canal during neck flexion, due to the growth imbalance of the vertebrae and dura mater rather than motor neuron disease that may occur at any site $(5,6)$.

Additional flexion view in routine cervical spine MRI is essential in case of suspected HD. The proposed MRI protocol includes routine cervical spine sequences in neutral position and additional scan with cervical spine flexion at $20^{\circ}$ to $40^{\circ}$ (7). The importance of additional flexion view is emphasized because the diagnosis of HD mainly relies on the characteristic image findings, including posterior dural anterior shift with loss of dural attachment and epidural space enlargement with flow void. Moreover, the narrowing of the arachnoid space is only stressed in flexion view in many cases (7). Although the suggested flexion angle is $20^{\circ}$ to $40^{\circ}$, the reported cases may vary, including mild flexion view of less than $20^{\circ}$. Insufficient flexion angle may hinder the timely diagnosis of HD, despite performing flexion cervical MRI (8), and may result in false-negative image finding. The previously proposed position was recommended before the surface coil, including neck coil, for cervical MRI was widely used. To the best of the author's knowledge, previous researchers have not focused on describing the limitation of neck coil for flexion MRI.

The current report presents a case of a 17-year-old young male newly diagnosed with HD, best detected by full cervical flexion scan in prone position.

\section{Case Presentation}

A 17-year-old young male was referred to the rehabilitation department of the current researchers for electro- 
physiological evaluation. He had progressive weakness of the right hand and hand tremor, which started about a year ago. He also reported intermittent hand tremor. No family history of neuromuscular or systemic disease was reported. Neurological examination showed subtle atrophy of the medial forearm muscle, ulnar forearm flexor, and wrist/finger extensor muscles and weakness during right wrist flexion/extension and right thumb extension. Right lateral forearm muscles, especially the brachioradialis muscle, were relatively spared. Grip strength was 19 $\mathrm{kg}$ on the right side and $30 \mathrm{~kg}$ on the left side, representing asymmetrical weakness. Power tested by manual muscle test was reduced with $\mathrm{F}+$ grade on right wrist flexion and $G$ grade throughout the right upper limb. Deep tendon reflexes were symmetrical yet decreased in upper extremities, and were normal in lower extremities. There was no fasciculation, cramps, sensory disturbances, such as hypoesthesia, paresthesia, and hyperalgesia on both upper and lower extremities. Pathologic reflex examinations, including Hoffmann sign, ankle clonus, and jaw jerk, were normal and showed no evidence of extrapyramidal involvements. Winging scapula was not observed on both sides. Routine blood analysis was within the normal range. Cerebrospinal fluid analysis was acellular without evidence of intrathecal immunoglobulin synthesis. Nerve conduction study showed no abnormalities, and electromyography showed denervation of the supraspinatus, infraspinatus, deltoid, triceps, biceps brachii, pronator teres, flexor carpi radialis and abductor pollicis longus on right side, extensor indicis profundus, and first dorsal interossei, bilaterally. Quantitative electromyography revealed large-amplitude and long-duration polyphasic motor unit action potentials on the examined muscles. Genetic analysis of amyotrophic lateral sclerosis-related genes (superoxide dismutase 1 [SOD1], Fused in Sarcoma [FUS], and C9orf72) and Survival Motor Neuron Protein 1 (SMN1) did not show any mutation. Plain radiography of the upper extremity and clavicular area showed no definitive abnormal structure. Magnetic Resonance Imaging was performed subsequently to evaluate the brachial plexus. In brachial plexus MRI, including neutral position cervical cord imaging, both sides of the brachial plexus showed signal intensity within the normal range without significant fascicular swelling. Significant intervertebral disc herniation and definitive cord-compressing lesion were not found.

After exclusion of brachial plexopathy, the flexion cervical MRI was taken under high clinical suspicion of HD. A 3T scanner (Verio, Siemens Healthcare, Erlangen, Germany) with 12-channel neck coil was used. The patient underwent MRI in neutral and flexion positions. For flexion MRI, the patient was asked to tuck his chin into his chest. In routine cervical MRI with neutral position, there was no sign of cord atrophy or edema (Figure 1A). The first attempt of flexion MRI was performed using a neck coil. With flexion position, the patient complained of aggravation of symptom, and obtaining optimized image with minimal motion artifact was not easy. Sagittal T2-weighted half-Fourier acquisition single-shot turbo spin-echo (HASTE) was obtained, and the flexion angle was only $10^{\circ}$. The image demonstrated hypotrophy of the cervical spinal cord at C5 - 6 with intramedullary edema (Figure 1B). However, anterior dural displacement was not depicted. The researchers found that even with pad underneath the cervical area and head, convenient support was not achieved for the patient. In addition, the full flexion of the neck was not attained because of the limited space and the nature of the customized cervical coil (round collar shaped with $19 \mathrm{~cm}$ diameter). Since HD was highly suspicious, optimized full flexion view was necessary. The second attempt was performed with 12-channel body coil in decubitus position to minimize the gap between the coil and the cervix. By this position, the cervical spine showed a $30^{\circ}$ flexion. Though the obtained sagittal T2-weighted HASTE image was unsatisfactory in image quality, approximately $2 \mathrm{~mm}$ anterior dural displacement was noted at C3-C6 (Figure 1C). For the third attempt, the patient was put in prone position with arms up with cervical full flexion until the chin touched the chest wall. In this position, the cervical spine was able to flex $40^{\circ}$. The detachment of the posterior dura from the lamina was definite from $\mathrm{C} 3$ to $\mathrm{T} 3$ with the largest diameter ( $6 \mathrm{~mm})$ at C5 - C6 level, and an engorged venous plexus was depicted with strong enhancement. The cervical cord was displaced anteriorly, and cord compression was noted (Figure 1D).

The commercialized wedge-shaped pad was applied under the tucked chin, and the pillow-shaped pad was applied under the arm. The 12-channel body coil was covered at the posterior aspect of the cervical spine (Figure 2). As a result, a diagnosis of HD was made.

\section{Discussion}

The current paper introduces a case of a 17-year-old young male with right-side upper weakness, identified as HD through multiple attempts of flexion cervical MRI. HD was not depicted in initial brachial plexus MRI, including neutrally positioned cervical cord MRI. The typical outstanding imaging findings were depicted only in prone position, which led to stable full flexion of the patient. In this patient, previous scan with flexion position and coil did not provide satisfactory image findings because of the following reasons: (1) Supine position with cervical flexion inside the neck coil-full flexion was limited by the relatively small space, and even with pad underneath the head and neck, the patient felt uncomfortable support, and flexion 

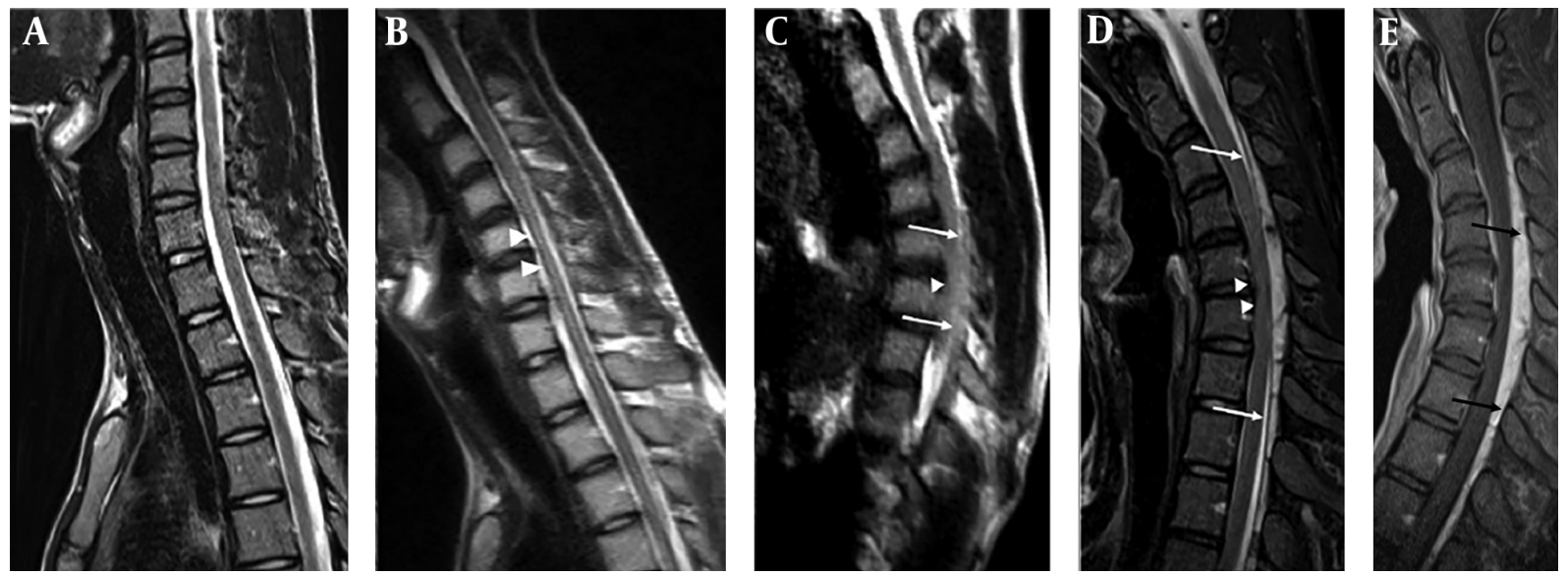

Figure 1. Serial cervical MRI of 17-year old male with Hirayama Disease. Neutral position, the sagittal T2-weighted MRI demonstrates no sign of cord atrophy or abnormal signal change (A). 1st flexion MRI trial using neck coil (flexion angle $=10$ degree), the sagittal T2-weighted half-Fourier acquisition single-shot turbo spin-echo (HASTE) image shows hypotrophy of cord at $\mathrm{C} 5 \sim 6$ (arrowheads) with edema (B). 2nd flexion MRI trial using body coil with decubitus position (flexion angle $=30$ degree), the sagittal T2-weighted HASTE image shows mild anterior displacement of posterior dura (arrows) at C3 6 (full level not shown in this image) with cord compression (arrowhead) (C). 3rd flexion MRI trial using body coil with prone position, T2-weighted image demonstrates definitive anterior dural displacement (arrows) at C3 T3 and cervical cord compression at C5 $\sim 6$ (arrowheads) (D). Note the enlarged epidural space with fluid void and strong enhancement (arrows) at enhanced fat suppressed T1-weighted image (E).

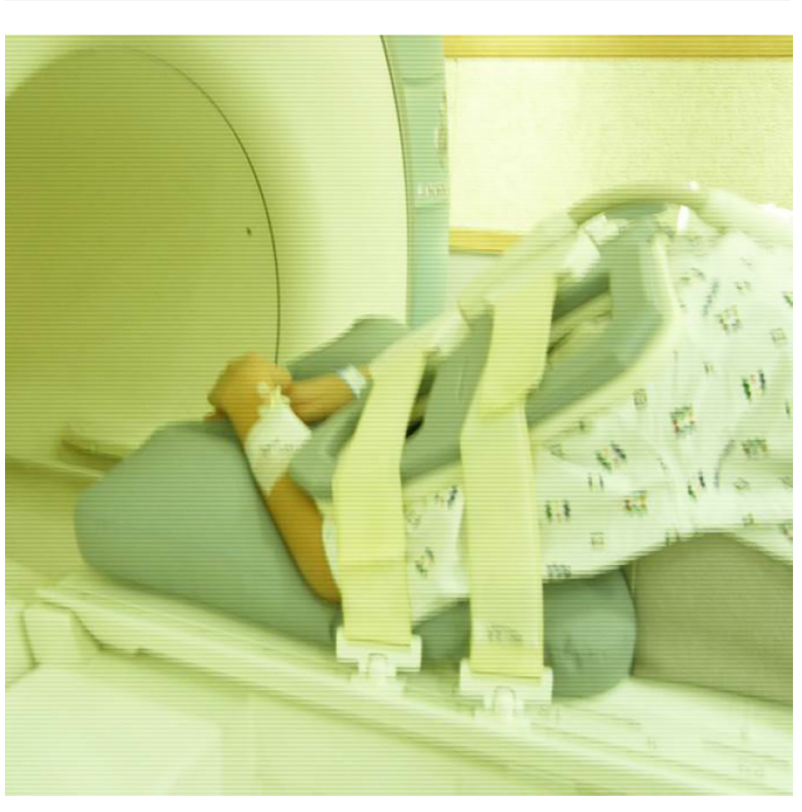

Figure 2. Picture of patient with prone position. The commercialized wedge shape pad was applied under the tucked chin and the pillow shape pad was applied under the arm. The 12-channel body coil was covered at the posterior aspect of cervical spine. Note that the most of the body surface was contacted with pad underneath.

position worsened the symptoms, causing motion artifact, and (2) with decubitus position with cervical flexion using body coil, adequate image quality was not obtained. In a previous report $(7,9,10)$, there have been image comparisons between the neutral position and flexion, and there have been image comparisons from patient to patient in a similar position; however, there have not been comparisons of images of HD relative to four different positions in one patient.

In the current case, full flexion and optimized image quality were obtained by the prone position using body coil. With most of the body surface supported by the pad, comfortable stabilization was possible. Cervical flexion in supine position is against the gravity, whereas the prone position was relatively free from gravity. The prone position for MRI diagnosis of HD has not been reported before. In addition, this position has a strong point, as it is easy and simple to achieve, and it can be done without a homebuilt pad. Instead, it can be done with a widely-used, commercialized, wedge-shaped pad underneath the chest, with the chin tucked in and a pillow-shaped pad underneath the arm. Using this position, the patient was able to flex his cervical area and obtain the ideal angle.

Even though HD is known to have benign and selflimiting course, accurate and timely diagnosis is essential for proper treatment. Early conservative treatment, including placement of the rigid cervical collar and preventing neck flexion, has been reported to significantly slow the progression and results in better functional outcomes $(2,11)$. Moreover, in case where progressive deterioration appears, the surgical approach could be considered in limited cases $(12,13)$.

The image findings of HD in neutral scan is known to show loss of attachment between the posterior dural sac and subjacent lamina to be the most effective findings for diagnosis of HD in neutral MRI, showing sensitivity and 
specificity of $93.5 \%$ and $98.0 \%$, respectively (14). However, in the current patient, such findings were not depicted in neutral scan, stressing the necessity of flexion image for imaging diagnosis of HD.

The characteristic finding of flexion scan of HD includes forward displacement of the posterior dura, posterior epidural space engorgement from the engorged venous plexus, and anterior cord displacement with cord compression with or without intramedullary edema $(6,9)$. The most noticeable feature in the present case was that all of the above-mentioned findings were only best and clearly depicted in full flexion with prone position at serial trial of flexion scan.

Some authors, including Hirayama and Tokumaru (9), described a specific position for flexion MRI, that is, to take cervical flexion as far forward as possible using pad, sponge, and pelvic wedge $(7,9,15)$. However, most researchers have not described in detail their MRI protocol, including the type of coil that was used. After dedicated surface coil has been applied in most musculoskeletal imaging, neck coil is widely used for cervical MRI. After the researchers' first attempt at using neck coil, they learned that the proposed position in previous reports was not feasible. Full flexion of the cervical spine was not attained because of the rigid collar shape with a diameter of $19 \mathrm{~cm}$, resulting in limited space. The cervical flexion angle in the current patient was only $10^{\circ}$. By this position, only cord atrophy and edema at the C5 - C6 level was depicted. The strengths of the current study include direct comparison of MR imaging obtained from different coils, which has not been introduced in other previous studies.

By serial flexion scan, the researchers found that the anterior dural displacement, which is known to be the most significant and important finding for the diagnosis of HD (9), may not be depicted in minimal flexion angle (first attempt), and the diameter and level increased as flexion angle increased. When the flexion angle increased from $30^{\circ}$ (second attempt) to $36^{\circ}$ (third attempt), the diameter of the shifted dura increased from $2 \mathrm{~mm}$ to $6 \mathrm{~mm}$ and the level of the shifted dura increased from C3 - C6 to C3 - T3. This is in accordance with a recent report by Kontzialis et al. (8), where they emphasized the importance of adequate flexion angle by introducing a case with failed expected dural anterior displacement at mild flexion scan. During their second attempt at tucking the chin, there was unequivocal anterior dural displacement. A previous multisite study reported the MRI findings of 21 patients with HD, where the false-negative rate was defined as without anterior dural shift at approximately 29\% (16). The authors assume one possible reason can be the fact that the angle of flexion was not controlled resulting in a possibility of inadequate flexion.

Although the suggested flexion angle is $20^{\circ}$ to $40^{\circ}$, one reason for the fact that full flexion position essentially did not get the limelight in many reported cases is that the dural displacement could be shown with insufficient flexion angle of less than $20^{\circ}$ in some cases. Thus, the current researchers propose to obtain additional full flexion scan, in case when insufficient flexion angle gives fewer information of a patient clinically highly suspicious of HD.

In summary, this study presents a case using collarshaped neck coil for cervical flexion MRI, and adequate flexion angle not able to be secured, resulting in insufficient information for imaging diagnosis of HD. By serial flexion scan, the researchers found that with insufficient flexion angle, dural displacement may not be evident. Furthermore, as the flexion angle increases, the diameter and level of the anterior dural displacement increases, emphasizing the necessity of full flexion imaging in patients clinically suspicious of HD. In such case, prone position may provide optimized flexion imaging.

\section{Footnotes}

Authors' Contributions: None declared.

Conflict of Interests: The authors declare that they had no conflicts of interests.

Ethical Approval: This case report was approved by the regional ethical review board, and informed consent was waived.

Financial Disclosure: None declared.

Funding/Support: This research was supported by a grant of the Korea Health Technology R\&D Project through the Korea Health Industry Development Institute (KHIDI), funded by the Ministry of Health and Welfare, Republic of Korea (grant number: HI15C1529).

\section{References}

1. Sobue I, Saito N, Iida M, Ando K. Juvenile type of distal and segmental muscular atrophy of upper extremities. Ann Neurol. 1978;3(5):429-32. doi: 10.1002/ana.410030512. [PubMed: 727722].

2. Tashiro K, Kikuchi S, Itoyama Y, Tokumaru Y, Sobue G, Mukai E, et al. Nationwide survey of juvenile muscular atrophy of distal upper extremity (Hirayama disease) in Japan. Amyotroph Lateral Scler. 2006;7(1):38-45. doi: 10.1080/14660820500396877. [PubMed: 16546758].

3. Hirayama K. Non-progressive juvenile spinal muscular atrophy of the distal upper limb (Hirayama's disease). Handbook of clinical neurology, diseases of the motor system. Amsterdam: Elsevier Science Publishers; 1991. p. 107-20.

4. Gourie-Devi M, Suresh TG, Shankar SK. Monomelic amyotrophy. Arch Neurol. 1984;41(4):388-94. [PubMed: 6703940].

5. Kikuchi S, Tashiro K, Kitagawa M, Iwasaki Y, Abe H. [A mechanism of juvenile muscular atrophy localized in the hand and forearm (Hirayama's disease)-flexion myelopathy with tight dural canal in flexion]. Rinsho Shinkeigaku. 1987;27(4):412-9. Japanese. [PubMed: 3621739]. 
6. Chen CJ, Chen CM, Wu CL, Ro LS, Chen ST, Lee TH. Hirayama disease: MR diagnosis. AJNR Am J Neuroradiol. 1998;19(2):365-8. doi: 10.1016/j.jocn.2014.11.025. [PubMed: 9504496].

7. Foster E, Tsang BK, Kam A, Storey E, Day B, Hill A. Hirayama disease. J Clin Neurosci. 2015;22(6):951-4. doi: 10.1016/j.jocn.2014.11.025. [PubMed: 25766368].

8. Kontzialis M, Yahyavi-Firouz-Abadi N, Zamora CA. Hirayama disease: The importance of adequate flexion MRI for diagnosis. Neurol Sci. 2015;36(9):1695-6. doi: 10.1007/s10072-015-2222-1. [PubMed: 25896623].

9. Hirayama K, Tokumaru Y. Cervical dural sac and spinal cord in juvenile muscular atrophy of distal upper extremity. Neurol ogy. 2000;54(10):1922-6. doi: 10.1007/s004150050514. [PubMed 10822430].

10. Schroder R, Keller E, Flacke S, Schmidt S, Pohl C, Klockgether T, et al. MRI findings in Hirayama's disease: Flexion-induced cervical myelopathy or intrinsic motor neuron disease? J Neurol. 1999;246(11):1069-74. [PubMed: 10631640].

11. Tokumaru Y, Hirayama K. [A cervical collar therapy for nonprogressive juvenile spinal muscular atrophy of the distal upper limb (Hirayama's disease)]. Rinsho Shinkeigaku. 1992;32(10):1102-6. Japanese. [PubMed: 1297554].
12. Tokumaru Y, Hirayama K. [Cervical collar therapy for juvenile muscular atrophy of distal upper extremity (Hirayama disease): results from 38 cases]. Rinsho Shinkeigaku. 2001;41(4-5):173-8. Japanese. [PubMed: 11676157].

13. Paredes I, Esteban J, Ramos A, Gonzalez P, Rivas JJ. A severe case of Hirayama disease successfully treated by anterior cervical fusion. J Neurosurg Spine. 2014;20(2):191-5. doi: 10.3171/2013.10.SPINE13508. [PubMed: 24286527].

14. Chen CJ, Hsu HL, Tseng YC, Lyu RK, Chen CM, Huang YC, et al. Hirayama flexion myelopathy: Neutral-position MR imaging findingsimportance of loss of attachment. Radiology. 2004;231(1):39-44. doi: 10.1148/radiol.2311030004. [PubMed: 15068939].

15. Lai V, Wong YC, Poon WL, Yuen MK, Fu YP, Wong OW. Forward shifting of posterior dural sac during flexion cervical magnetic resonance imaging in Hirayama disease: An initial study on normal subjects compared to patients with Hirayama disease. Eur J Radiol. 2011;80(3):724-8. doi: 10.1016/j.ejrad.2010.07.021. [PubMed: 20727701].

16. Lehman VT, Luetmer PH, Sorenson EJ, Carter RE, Gupta V, Fletcher GP, et al. Cervical spine MR imaging findings of patients with Hirayama disease in North America: A multisite study. AJNR Am J Neuroradiol. 2013;34(2):451-6. doi: 10.3174/ajnr.A3277. [PubMed: 22878010]. 